\title{
Leveque-Type Similarity Transformation for a Thermally Developing Viscous Dissipative Flow in a Parallel Plate Channel
}

\author{
Gooi Mee Chen*, Yew Hau Yip \\ Centre for Advanced Mechanical and Green Technology, Faculty of Engineering and Technology, Multimedia University, \\ Jalan Ayer Keroh Lama, 75450 Bukit Beruang, Melaka, Malaysia
}

Corresponding Author Email: gmchen@mmu.edu.my

https://doi.org/10.18280/ijht.390440

Received: 23 May 2020

Accepted: 22 May 2021

\section{Keywords: \\ Leveque-type similarity transformation, thermally developing, forced convection,} viscous dissipation

\begin{abstract}
Compared to the existing more elaborate eigenvalues-eigenfunction analytical solution where the solution of a thermally developing forced convection problem converges very slowly at the beginning of thermal entrant region, Leveque-type similarity transformation method provides a more convenient way to look into the insights of the problem. Assuming that the wall heat flux and viscous dissipation only has an effect within the thin thermal boundary layer at the beginning of the thermal entrance region, this study intends to solve the governing thermal energy equation for a thermally developing flow in a parallel plate channel, subjected to uniform heat flux, by means of Leveque-type similarity transformation. The resulting ordinary differential equation, is subsequently solved by a fourth order Runge Kutta method. A comparison of the Nusselt number along the axial direction, at the beginning of the thermally developing region with the literature, reveals less than $10 \%$ discrepancy for Brinkman number less than one, which is a commonly acceptable range for practical applications. Although its accuracy depletes downstream the channel, similarity transformation provides sufficiently accurate temperature distribution, and captures the heat transfer insights for a thermally developing viscous dissipative forced convection.
\end{abstract}

\section{INTRODUCTION}

Laminar forced convective heat transfer commonly encountered in heating and cooling related appliances such as heat exchanger and heat sink, usually has significant thermal entrance effects in view of its relatively larger entrance length as compared to heat transfer in turbulent flow. On the other hand, the growth in applications of microscale devices in areas like biological instrumentation, electronics cooling and so on has highlighted the significance of viscous dissipation on moderate to high-speed flows in microchannels.

A number of studies and research has been focused on laminar forced convection heat transfer at entrance region. One of the pioneering work solved the forced convection of laminar flow in a circular tube under the isoflux conditions analytically by using a set of eigenvalues and eigenfunctions [1]. The study was extended to look into a similar problem for parallel plate geometry but applied to ducts with unequal wall temperatures [2, 3]. General methods, namely separation of variables and the Leveque-type similarity transformation were used to derive analytical solutions for the temperature distributions of a thermally developing flow within parallel plates, subjected to constant heat flux conditions [4]. The analytical solution obtained in the form of infinite series, in terms of eigenfunctions and eigenvalues, however converges very slowly and hence an accurate solution requires an exceptionally large number of eigenvalues at the beginning of the thermal entrance region. Apparently, the refined Levequetype of similarity solution provides a more convenient way to determine the temperature field and Nusselt number, without losing marked accuracy at the beginning of thermal entrance region [5]. In another study, a numerical method is applied to investigate forced convection for a mist flow in a concentric annuli in the thermal entrance region, revealing the effect of a few pertinent parameters in liquid loading parameter, the heat sink parameter, the wall superheat parameter, and the radius ratio on heat transfer characteristic in mist flow [6]. Miniaturization of heat sinks intensify research in thermally developing laminar forced convection in recent decades. In a related study on Graetz problem, which specifies constant but unequal wall temperatures, it was solved numerically using Mathematica [7]. Heat transfer of laminar flow in the thermal entrance region within rectangular microchannels were investigated in various aspect ratios and solved using computational fluid dynamics (CFD) [8, 9]. The study remarked a rapid decrease in Nusselt number as the flow approaches fully developed region. The afore-mentioned studies however did not consider the effect of heat generation on the temperature field in forced convective heat transfer. The emergence of microscale ducts led to more studies on viscous dissipation effects in thermally developing flows, either by separation of variables or numerical method. An investigation on forced convective heat transfer of Poiseuille flow in parallel plates, considering the viscous dissipation effect for the thermally developing case was solved numerically [10], while a thermally developing temperature profile in circular ducts and parallel plates was studied numerically, by taking into account of the viscous dissipation where the ducts were subjected to uniform wall temperature [11]. Forced convective thermally developing flow in a circular duct with the viscous 
dissipation effects under uniform wall temperature boundary condition method was later on tackled, by employing separation of variables [12]. Another study reported an analytical solution obtained by Laplace transform method [13] for different thermal boundary condition namely axiallyvarying wall heat flux condition. SAR (Successive Accelerated Replacement) was implemented to provide the numerical solutions for laminar forced convection heat transfer, of a thermally developing flow within asymmetrically heated parallel plates [14]. Their work was furthered by taking into account of the effects of viscous dissipation [15]. In another reported study, heat transfer in a thermally developing microscale tube was investigated analytically in the slip flow region, taking into account of the effects of axial conduction, viscous dissipation and rarefaction [16]. The local temperature field was obtained in terms of Peclet number, Knudsen number, Brinkman number and a parameter affecting the temperature jump. Nusselt number was found to decrease with increasing Knudsen number due to the effect of temperature jump. The effect of viscous dissipation on a laminar flow, in the thermal entrance region of a rectangular channel with rounded corners, subject to uniform wall temperature was investigated [17]. New correlations were developed in the study to predict the Poiseuille number and Nusselt number. The aforementioned studies on forced convection of thermally developing flow in the presence of viscous dissipation are usually analysed, either analytically with the use of a huge number of eigenvalues or through numerical methods to solve the governing partial differential equations at the entrance region. This study will look into an alternative way to determine the heat transfer coefficient of a laminar viscous dissipative flow at the beginning of the thermal entrance region, via Leveque-type similarity transformation, which is less studied in such aspects.

This work contains three subsequent parts, namely Part 2. Problem Formulation, Part 3. Results and Discussion, and lastly Part 4 on Conclusions.

\section{PROBLEM FORMULATION}

Consider an infinitely long parallel plate channel with height $2 H$, where a constant heat flux $q_{w}$ "is applied to the channel wall as shown in Figure 1. A hydrodynamically fully developed flow and a uniform inlet fluid temperature $T_{o}$, is assumed. $\mathrm{x}$ axis is defined along the bottom plate in the flow direction while $y$ axis originates from the leading edge of bottom plate in the transverse direction. Due to symmetry, only half of the channel is considered in the subsequent analysis.

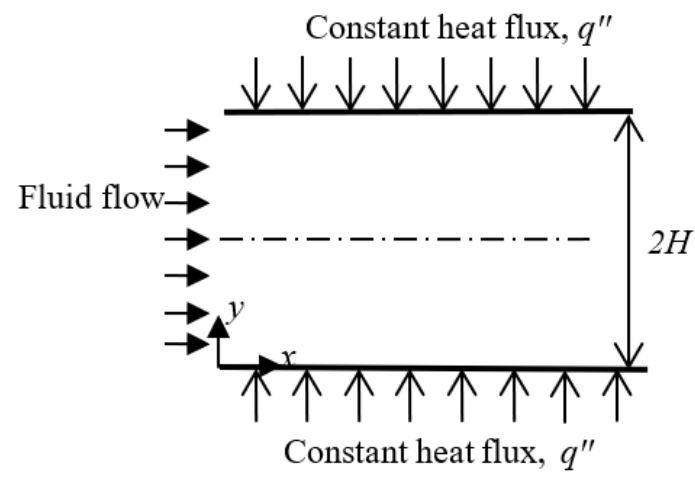

Figure 1. Schematic diagram of the problem
Taking into account of viscous dissipation, the governing thermal energy equation assuming a hydrodynamically fully developed flow can be expressed as [10]:

$$
u \frac{\partial \mathrm{T}}{\partial \mathrm{x}}=\frac{\mathrm{k}}{\rho c_{p}}\left(\frac{\partial^{2} T}{\partial y^{2}}\right)+\frac{\mu}{\rho c_{p}}\left(\frac{d u}{d y}\right)^{2}
$$

where, the Poiseuille flow is described as:

$$
u(y)=\frac{3}{2} u_{m}\left(\frac{y}{H}\right)\left[2-\left(\frac{y}{H}\right)\right]
$$

The corresponding thermal boundary conditions are:

$$
\begin{gathered}
\text { B.C. } 1: x=0, T=T_{o}, \\
\text { B.C. } 2: y=0,-k \frac{\partial T}{\partial y}=q^{\prime \prime}, \\
\text { B.C. } 3: y=H, \frac{\partial T}{\partial y}=0,
\end{gathered}
$$

By defining

$$
Y=\frac{y}{H}, X=\frac{x / H}{P e}, P e=\frac{u_{m} H}{\alpha}, B r=\frac{\mu u_{m}^{2}}{H q^{\prime \prime}}, \theta=\frac{k\left(T-T_{o}\right)}{H q^{\prime \prime}}
$$

and assuming that the thermal boundary layer is much thinner than the characteristic height of the parallel plate channel, the velocity field is simplified as,

$$
\frac{u}{u_{m}} \approx 3 \mathrm{Y}
$$

Eq. (1) may be recast as,

$$
3 Y \frac{\partial \theta}{\partial \mathrm{X}}=\frac{\partial^{2} \theta}{\partial Y^{2}}+9 B r
$$

along with the thermal boundary conditions specified as,

$$
\begin{gathered}
X=0, \theta=0 \\
Y=0, \frac{\partial \theta}{\partial Y}=-1 \\
Y \rightarrow \infty, \frac{\partial \theta}{\partial Y}=0
\end{gathered}
$$

Following the assumption made on the velocity, the resulting viscous dissipation term in Eq. (4) is a constant and according to the method outlined [18], with the understanding that the heat generation term diminishes with increasing $Y$, viscous dissipation effect is only restricted within a thin thermal boundary layer.

A new parameter [18],

$$
\varphi=\frac{d \theta}{d Y}
$$

is defined to represent the dimensionless heat flux. Eq. (4) may be transformed to

$$
3 Y \frac{\partial \varphi}{\partial X}=-\frac{1}{Y} \frac{\partial \varphi}{\partial Y}+\frac{\partial^{2} \varphi}{\partial Y^{2}}-\frac{9 B r}{Y}
$$


alongside the boundary conditions stated as

$$
\begin{gathered}
X=0, \theta=0 \\
Y=0, \varphi=-1 \\
Y \rightarrow \infty, \varphi=0
\end{gathered}
$$

In order to solve Eq. (6) by means of similarity transformation, applied to the beginning of thermally developing region where the heat flux and viscous dissipation only have an effect on a thin boundary layer, construed as much smaller than half the height of the parallel plate channel $H$, a similarity variable, $\eta$ is next introduced as

$$
\eta=\frac{Y}{s(X)}
$$

Eq. (6) may hence be transformed to

$$
\frac{\partial^{2} \varphi}{\partial \eta^{2}}+\frac{\partial \varphi}{\partial \eta}\left(-\frac{1}{\eta}-\frac{9 B r s(X)}{\eta}+3 \eta^{2} s(X)^{2} s(X)^{\prime}\right)=0
$$

By letting $s(X)=(3 X)^{\frac{1}{3}}$, and similarity variable, $\eta=\frac{Y}{(3 X)^{\frac{1}{3}}}$, Eq. (7) may be simplified to an O.D.E. in the form,

$$
\frac{\partial^{2} \varphi}{\partial \eta^{2}}+\frac{\partial \varphi}{\partial \eta}\left(-\frac{1}{\eta}+3 \eta^{2}\right)=\frac{K}{\eta}
$$

In Eq. (9), $X$ is assumed as a parameter embedded in $K=$ $9 \operatorname{Br}(3 X)^{1 / 3}$, which incorporates the viscous dissipation effect. Eq. (8) is subjected to two boundary conditions designated as

$$
\begin{aligned}
& \eta=0, \varphi=-1 \\
& \eta \rightarrow \infty, \varphi=0
\end{aligned}
$$

Similarity method outlined in Ref. [18] has been extended to viscous dissipative flow in this study and successfully converts the governing thermal energy equation to an O.D.E. with the assumption that the wall heat flux and viscous dissipation only has an effect on the heat transfer within the thin thermal boundary layer at the beginning of the thermal entrance region.

Eq. (9) is solved numerically by employing Runge Kutta fourth order algorithm, where at the isoflux and adiabatic boundaries, $\eta$ are taken as $1 \times 10^{-5}$ and 1.5 respectively. Eq. (9) is first transformed into first order O.D.E. before applying the Runge Kutta fourth order algorithm. The numerical data obtained is then recast as a $6^{\text {th }}$ order polynomial function while $\theta$ at $Y$ is obtained by integrating $\varphi$ numerically

$$
\theta(Y)=(3 X)^{1 / 3} \int_{\infty}^{\eta} \varphi d \eta
$$

The Nusselt number at a specified $X$ is obtained by further computing the bulk mean temperature from $\theta$ relative to $\theta_{w}$.

$$
N u=\frac{2}{\theta_{B}-\theta_{w}}
$$

\section{RESULTS AND DISCUSSION}

\subsection{Verification of results}

In order to verify the accuracy of the results using the present methodology, $N u$ computed in this study is compared to the literature whereby the $N u$ in the reported study [19] was solved by using an infinite series in terms of eigenvalues and eigenfunctions. For comparison purpose, the $\mathrm{Nu}$ in the aforementioned study [19] is recast and reduced to the limiting case for clear fluid. The $N u$ presented in Table 1, as a function of dimensionless axial distance, $X$, and with Brinkman number, $\mathrm{Br}$ as the parameter shows an excellent agreement between the present work and the literature at the beginning of the thermal entrance region, and up to $X=0.08$. From Table 1 , the discrepancy from the literature is $-2.06 \%$ for $B r=0.1$ and -8 . $74 \%$, for $B r=1$ respectively. The accuracy depletes moving downstream and with increasing Brinkman number, as shown in Table 1 . The fully developed distance based on $X$ defined in this study is estimated to be about $X=0.8$ in the literature. Therefore, applying similarity transformation to the governing thermal energy equation, followed by deploying a fourth order Runge Kutta numerical method to solve the resulting O.D.E., is able to estimate the temperature field and $N u$ for a viscous dissipative flow with an error of less than $10 \%$ for Br spanning between 0 and 1 , an acceptable range in the practical application, and up to $1 / 10$ of the thermally developing region, at $X=0.08$. In view of its simplicity and insights to the problem, similarity transformation is a viable solution to this slowly converging problem at the beginning of the thermally developing region.

Table 1. Nu comparison with the literature

\begin{tabular}{cccc}
\hline$X$ & $B r$ & $N u$ (present study) & $N u[19]$ \\
\hline 0.00016 & 0.1 & 33.70 & 33.56 \\
0.0008 & 0.1 & 19.42 & 19.28 \\
0.0016 & 0.1 & 15.30 & 15.20 \\
0.008 & 0.1 & 8.81 & 8.77 \\
0.016 & 0.1 & 6.98 & 6.98 \\
0.08 & 0.1 & 4.28 & 4.37 \\
0.8 (F.D.) & 0.1 & & 3.553 \\
0.00016 & 1 & 26.80 & 26.70 \\
0.0008 & 1 & 13.60 & 13.68 \\
0.0016 & 1 & 10.00 & 10.16 \\
0.008 & 1 & 4.77 & 5.04 \\
0.016 & 1 & 3.46 & 3.75 \\
0.08 & 1 & 1.88 & 2.06 \\
0.8 (F.D.) & 1 & & 1.59 \\
\hline
\end{tabular}

\subsection{Discussion}

The dimensionless heat flux variation with $Y$ is depicted in Figure 2, with and without viscous dissipation respectively, showing self-similar pattern moving downstream the channel. Notably, heat generation in the form of viscous dissipation causes an increase in heat flux in the proximity of the wall and distorts the self-similar features as $X$ goes up. Figure 3(a) depicts that the thermal boundary layer where the heat flux penetrates thickens slightly due to viscous dissipation, not so significantly as compared to Figure 3(b) where $\mathrm{Br}$ is raised to 1. The parallel curves in the vicinity of the wall indicates the wall heat flux diffusion effect with increasing $\mathrm{Br}$, the curves display a larger gradient moving away from the wall before diminishing towards the centerline, highlighting the presence of higher viscous dissipation. As the thermal boundary 
thickness grows with increasing $B r$, but constrained by the assumption that $H$ should be much larger than this thickness, we expect the accuracy of the results to drop.

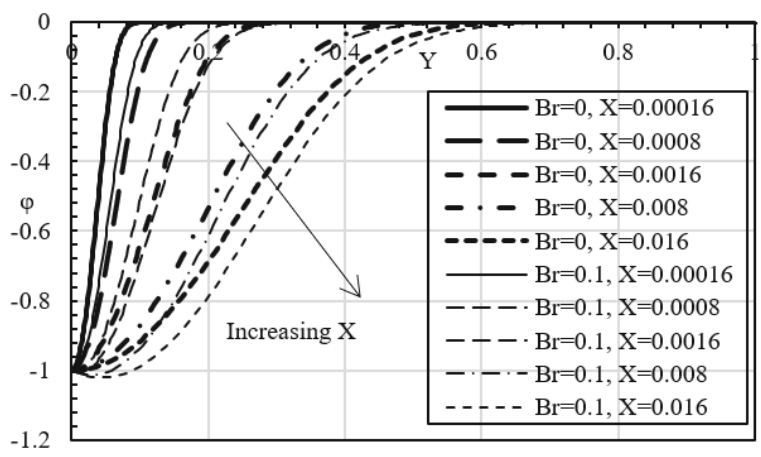

Figure 2. Dimensionless heat flux variation in the transverse direction at different $X$ for $B r=0$ and $B r=0.1$

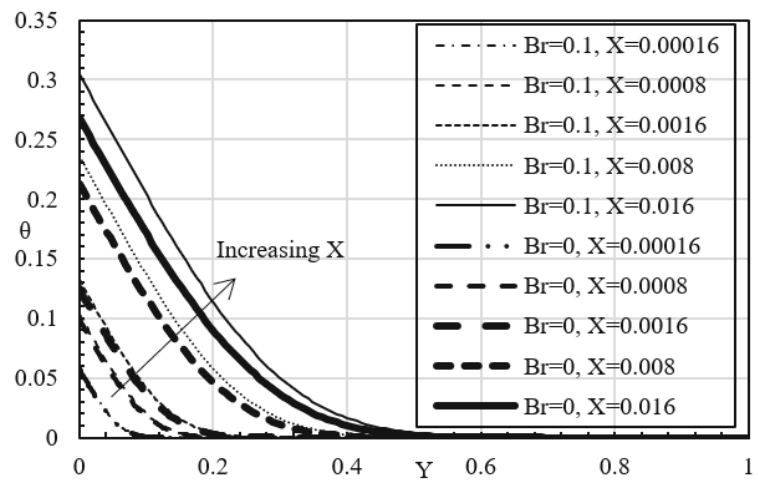

(a) $B r=0.1$ and $B r=0$ up to $X=0.016$

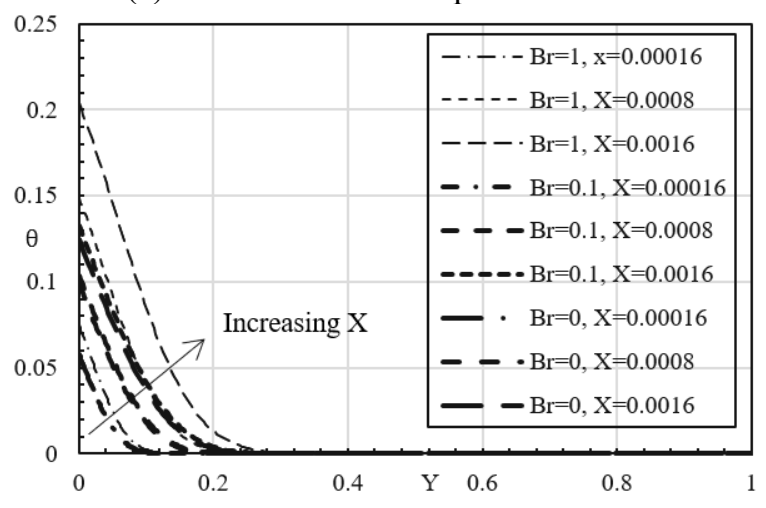

(b) $B r=1, B r=0.1$ and $B r=0$ up to $X=0.0016$

Figure 3. Dimensionless temperature field along the transverse direction at different $X$

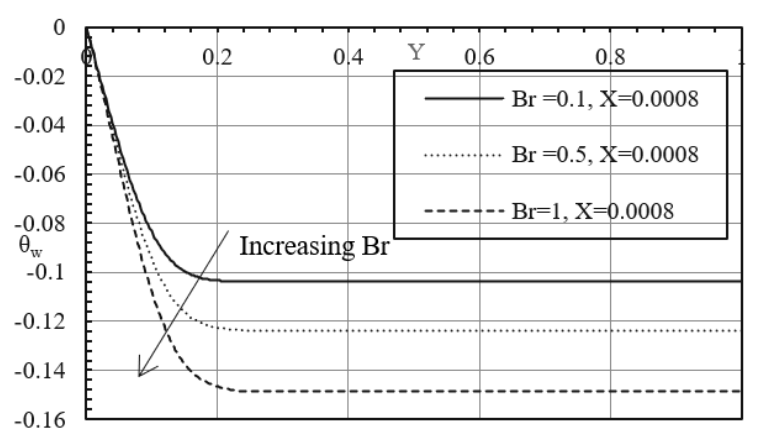

Figure 4. Dimensionless temperature distributions (Relative to wall temperature) along $Y$ at $X=0.0008$
Figure 4 shows appreciable change in temperature relative to the wall temperature as $\mathrm{Br}$ increases to 1, and reveals the increase in boundary layer thickness. It is worth mentioning that at $X=0.0008$, the effect of viscous dissipation is restricted to thin region next to the wall. From the boundary layer thickness, it can be inferred that the thermal entrance length for a thermally developing flow with more intense heat generation would be smaller, considering that thermally fully developed profile in an internal flow is achieved when the thermal boundary layer occupies half the height of the channel.

Figure 5 depicts $N u$ variation with $X$, the agreement is excellent for $B r=0.1$. For $B r=1$, the $N u$ in this study still concurs reasonably well with the $N u$ in the literature up to $X=0.08$, the underestimation in $N u$ with increasing $X$ is mainly due to linear velocity pattern assumed, which becomes erroneous as the thermal boundary layer penetrates further above the wall. The viscous dissipation, in turn would affect the accuracy of the results adversely as the thermal boundary layer thickens, as a result of the less accurate velocity profile within the not so thin boundary layer. The diminishing viscous dissipation generation moving away from the wall is not sufficiently represented within the thicker boundary layer, hence causing an overestimation in the heat generation and temperature field which renders a smaller $N u$. This effect is particularly obvious towards $X=0.1$ in Figure 5. Nonetheless, the results in this study are acceptable up to $X=0.08$, as verified in Section 3.1. The underestimation in $N u$ is due in part to the uniform viscous dissipation assumed in the boundary layer.

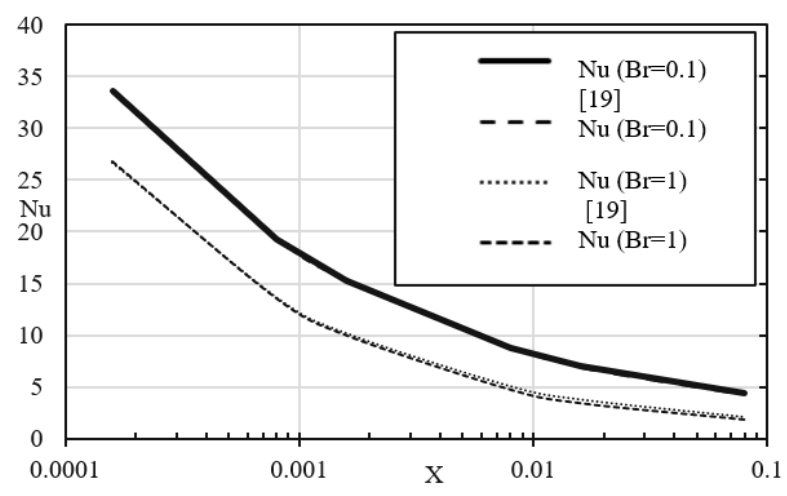

Figure 5. $N u$ variation along $X$

\section{CONCLUSIONS}

Leveque-type similarity transformation successfully converts the thermal energy equation for a viscous dissipative thermally developing force convection, subjected to constant heat flux, from a P.D.E. to an O.D.E., by defining the heat flux as self-similar function and a similarity variable assuming a much thinner thermal boundary layer compared to the characteristic height of the parallel plate channel, as well as $X$ as a parameter in the transformed equation for Brinkman number spanning between 0 and 1 . The resulting O.D.E. is then solved by Runge Kutta fourth order method. Results compared to the literature solved in terms of a series of eigenvalues and eigenfunctions at the very beginning of the thermal entrance region shows good agreement. Constrained by the assumptions that requires a thin thermal boundary layer, the solution produces larger discrepancy, moving downstream the channel and increasing $B r$. For $B r$ spanning between 0 and 
1 , this method is able to yield Nusselt numbers with errors less than $10 \%$ for $B r=1$, up to $1 / 10$ of the fully developed length, which is the region that sees the most change in the $N u$. Conclusively, Leveque-type similarity transformation on the governing thermal equation that bears a heat generation term for a thermally developing flow is able to produce results within reasonable accuracy at the very beginning of the thermally developing region, constraint by the thickness of the thermal boundary layer. In future, the coupled effects of the hydrodynamics development may be looked into.

\section{ACKNOWLEDGMENT}

This work is supported financially by the Ministry of Education Malaysia under research grant FRGS/1/2019/TK03/MMU/02/01.

\section{REFERENCES}

[1] Siegel, R., Sparrow, E.M., Hallman, T.M. (1958). Steady laminar heat transfer in a circular tube with prescribed wall heat flux. Applied Scientific Research, Section A, 7(5): 386-392. https://doi.org/10.1007/BF03184999

[2] Cess, R.D., Shaffer, E.C. (1959). Heat transfer to laminar flow between parallel plates with a prescribed wall heat flux. Applied Scientific Research, Section A, 8(1): 339344. https://doi.org/10.1007/BF00411758

[3] Hatton, A.P., Turton, J.S. (1962). Heat transfer in the thermal entry length with laminar flow between parallel walls at unequal temperatures. International Journal of Heat and Mass Transfer, 5(7): 673-679. https://doi.org/10.1016/0017-9310(62)90090-X

[4] Shah, R.K., London, A.L. (1978). Laminar Flow Forced Convection in Ducts: A Source Book for Compact Heat Exchanger Analytical Data, Academic Press, London.

[5] Worsøe-Schmidt, P.M. (1967). Heat transfer in the thermal entrance region of circular tubes and annular passages with fully developed laminar flow. International Journal of Heat and Mass Transfer, 10(4): 541-551. https://doi.org/10.1016/0017-9310(67)90173-1

[6] Hwang, T.H., Cheng, P., Lin, J.K. (1990). Heat transfer of laminar mist flow in conncentric annuli. Numerical Heat Transfer, Part A: Applications. 18(2): 243-258. https://doi.org/10.1080/10407789008944793

[7] Mitrović, J., Maletić, B., Bačlić, B.S. (2006). Some peculiarities of the asymmetric Graetz problem. International Journal of Engineering Science, 44(7): 436455. https://doi.org/10.1016/j.ijengsci.2006.02.003

[8] Lee, P.S., Garimella, S.V, Liu, D. (2005). Investigation of heat transfer in rectangular microchannels, International Journal of Heat and Mass Transfer, 48(9): 1688-1704.

https://doi.org/10.1016/j.ijheatmasstransfer.2004.11.019

[9] Lee, P.S., Garimella, S.V. (2006). Thermally developing flow and heat transfer in rectangular microchannels of different aspect ratios. International Journal of Heat and Mass $\quad$ Transfer, 49(17): 3060-3067. https://doi.org/10.1016/j.ijheatmasstransfer.2006.02.011

[10] Aydın, O., Avcı, M. (2006). Viscous-dissipation effects on the heat transfer in a Poiseuille flow. Applied Energy, 83(5):

495-512.

[11] Jambal, O., Shigechi, T., Davaa, G., Momoki, S. (2005). Effects of viscous dissipation and fluid axial heat conduction on heat transfer for non-Newtonian fluids in ducts with uniform wall temperature: Part II. annular ducts. International Communications in Heat and Mass Transfer, $\quad 32(9)$ : 1174-1183. https://doi.org/10.1016/j.icheatmasstransfer.2005.07.00 3

[12] Barletta, A., Magyari, E. (2006). Thermal entrance heat transfer of an adiabatically prepared fluid with viscous dissipation in a tube with isothermal wall. Journal of Heat Transfer, $\quad 128(11)$ : https://doi.org/10.1115/1.2352784

[13] Barletta, A., Magyari, E. (2007). Forced convection with viscous dissipation in the thermal entrance region of a circular duct with prescribed wall heat flux. International Journal of Heat and Mass Transfer, 50(1): 26-35. https://doi.org/10.1016/j.ijheatmasstransfer.2006.06.036

[14] Repaka, R., Satyamurty, V.V. (2010). Local and average heat transfer in the thermally developing region of an asymmetrically heated channel. International Journal of Heat and Mass Transfer, 53(9): 1654-1665. https://doi.org/10.1016/j.ijheatmasstransfer.2010.01.024

[15] Repaka, R., Satyamurty, V.V. (2013). Effect of viscous dissipation on forced convection heat transfer in parallel plate channels with asymmetric boundary conditions. ASME International Mechanical Engineering Congress and Exposition, IMECE2013-63112. https://doi.org/10.1115/IMECE2013-63112

[16] Barişik, M., Yazicioğlu, A.G., Çetin, B., Kakaç, S. (2015). Analytical solution of thermally developing microtube heat transfer including axial conduction, viscous dissipation, and rarefaction effects. International Communications in Heat and Mass Transfer, 67: 81-88. https://doi.org/10.1016/j.icheatmasstransfer.2015.05.00 4

[17] Suzzi, N., Lorenzini, M. (2019). Viscous heating of a laminar flow in the thermal entrance region of a rectangular channel with rounded corners and uniform wall temperature. International Journal of Thermal Sciences, 145: 106032 . https://doi.org/10.1016/j.ijthermalsci.2019.106032

[18] Deen, W.M. (2012). Analysis of Transport Phenomena. Oxford University Press, Oxford.

[19] Haji-Sheikh, A., Sparrow, E.M., Minkowycz, W.J. (2005). Heat transfer to flow through porous passages using extended weighted residuals method - A Green's Function Solution. International Journal of Heat and Mass Transfer, 48(7): 1330-1349. https://doi.org/10.1016/j.ijheatmasstransfer.2004.10.002

\section{NOMENCLATURE}

$\mathrm{Br}$

$\mathrm{C}_{\mathrm{p}}$

$\mathrm{H}$

$\mathrm{k}$

$\mathrm{Nu}$

$\mathrm{Pe}$

$q^{\prime \prime}$

$\mathrm{T}$

$\mathrm{u}$

$\mathrm{x}$
Brinkman number, defined in Eq. (4) specific heat capacity, $\mathrm{J} / \mathrm{kg} \cdot \mathrm{K}$ half of the height of microchannel (m) thermal conductivity, $\mathrm{W} / \mathrm{m} \cdot \mathrm{K}$ Nusselt number, defined in Eq. (12) Peclet number heat flux, $\mathrm{W} / \mathrm{m}^{2}$ fluid temperature, $\mathrm{K}$ axial velocity, $\mathrm{m} / \mathrm{s}$ axial distance, $\mathrm{m}$ 
dimensionless axial distance, defined in Eq

(5a)

vertical distance from the lower wall (m)

dimensionless vertical distance, defined in Eq. (4) $\theta$

$\mu$

$\rho$

$\varphi$

\section{Subscripts}

(1)

$\rho$

dimensionless temperature, defined in Eq.

(4)

viscosity, $\mathrm{Pa} \cdot \mathrm{s}$

density, $\mathrm{kg} / \mathrm{m}^{3}$

dimensionless heat flux, defined in Eq. (6)

\section{Greek symbols}

$\begin{array}{ll}b & \text { bulk mean } \\ \mathrm{o} & \text { initial } \\ w & \text { wall }\end{array}$

thermal diffusivity, $\mathrm{m}^{2} / \mathrm{s}$

$\eta \quad \begin{aligned} & \text { similarity dimensionless parameter, defined } \\ & \text { in Eq. (8) }\end{aligned}$

$\eta \quad \begin{aligned} & \text { similarity dimensionless parameter, defined } \\ & \text { in Eq. (8) }\end{aligned}$

$\alpha$ initial
wall 